\title{
Kikuchi-Fujimoto Diseasae: An Important Differential of Tuberculous Lymphadenitis
}

\author{
Shaheen Sultan, Hina Mushtaq and Abdul Manan \\ Department of Medicine, Liaquat National Hospital, Karachi, Pakistan
}

\begin{abstract}
Kikuchi-Fujimoto disease, also called necrotising histiocytic lymphadenitis, is a rare disease of usually benign outcome. A case of a 21-year female is reported here. She presented with high grade fever and cervical lymphadenopathy. She was started on anti-tubercular treatment (ATT) with suspicion of tuberculous lymphadenitis as she had very strong history of tubercular (TB) contact. Kikuchi-Fujimoto disease was diagnosed after cervical lymph node biopsy, thus ATT was stopped. She was treated with oral steroids and complete recovery was made. Of significant note, two of patient's siblings were treated for pulmonary TB, because of which she had strong history of direct TB contact. Although a few cases of Kikuchi-Fujimoto disease have been reported in Pakistan, this is first case being reported in setting of very strong positive history of TB contact.
\end{abstract}

Key Words: Lymphadenitis, Tuberculosis, Kikuchi-Fujimoto disease.

How to cite this article: Sultan S, Mushtaq H, Manan A. Kikuchi-Fujimoto Diseasae: An Important Differential of Tuberculous Lymphadenitis. J Coll Physicians Surg Pak 2020; 30(09):987-988.

\section{INTRODUCTION}

Kikuchi-Fujimoto disease (KFD), also known as histiocyticnecrotising lymphadenitis, is a rare, self-limiting condition characterised by regional lymphadenopathy, fever, night sweat, and upper respiratory symptoms. Exact etiology is unknown; however, a viral or autoimmune pathogenesis has been suggested. Diagnosis is based on histolopathologic characteristics noted on excisional biopsy. A majority of cases have a benign course; KFD rarely results in death. ${ }^{1}$

Recognition of KFD is essential because it can easily be mistaken for tuberculosis (TB), lymphoma and adenocarcinoma or at times with lymphadenitis associated with systemic lupus erythematosus (SLE). ${ }^{2}$ Here, we report a case of patient who was initially misdiagnosed as TB and was then treated for KFD.

\section{CASE REPORT}

A 21-year female, unmarried with no known comorbids, resident of Karachi, having no addiction and was on no medications, presented with high grade fever for two weeks. Her temperature was documented up to $101^{\circ} \mathrm{F}$, intermittent in nature with no specific pattern and with no other systemic manifestations, including sweating or weight loss.

Correspondence to: Dr. Shaheen Sultan, Department of Medicine, Liaquat National Hospital, Karachi, Pakistan E-mail: shaheensultan51@gmail.com

Received: September 05, 2018; Revised: January 29, 2020; Accepted: July 06, 2020

DOI: https://doi.org/10.29271/jcpsp.2020.09.987
There was no history of pet or bird exposure. Her two siblings had been treated for pulmonary TB. On examination, she was lethargic with temperature of $101^{\circ} \mathrm{F}$; otherwise, she was stable and rest of physical examination was unremarkable. Blood tests revealed raised total leukocyte count with normal differential count. Electrocardiogram and chest radiographs were normal. Blood, urine and stool cultures were initially negative. Sputum cultures grew respiratory tract flora and were negative for acid-fast bacilli. Work-up for malaria and dengue was negative. After initial work-up, she was started on broad spectrum antibiotics, while final results of cultures were awaited, which were negative finally for any bacterial growth. Her fever did not respond. The only significant positive finding in her laboratory work-up was raised erythrocyte sedimentation rate (ESR). On clinical grounds, she was started on anti-tubercular treatment(ATT).

After 15 days of therapy with ATT, her fever remained same. On re-examination, there was a single cervical lymph node found, which was not present previously. It was around $3 \times 3$ $\mathrm{cm}$, non-tender, mobile, smooth in contours, soft in consistency with no discharge from it. Lymph node biopsy was done. She was continued with ATT till biopsy results. Her fever did not subside. Biopsy results showed histiocytic necrotising lymphadenitis consistent with KFD. ATT was stopped and she was started on oral steroids. After two weeks of therapy with oral steroids, her fever resolved and lymph node regressed in size. She recovered successfully.

\section{DISCUSSION}

KFD is a rare condition of benign outcome. The disease was first described by Kikuchi and Fujimoto in Japan. ${ }^{3}$ This disease has 
predilection for Asian women between the ages of 20 and 35 years. ${ }^{4}$ However, new cases of KFD have also been reported in non-Asian ethnicity and young age groups. ${ }^{5}$ Cases in pediatrics and pregnant ladies have also been reported. Its etiology has not yet been fully determined; however, it is believed that it may be of viral origin, among which, Epstein-Barrvirus (EBV), human herpesvirus 6 (HHV6) and HSV8 have been suggested. ${ }^{2}$ An autoimmune etiology is also likely as it has been reported in association with $\mathrm{SLE}^{5}$ In some studies, association with Sjögren's syndrome and other autoimmune diseases has been found. ${ }^{6}$ Review of reported cases showed that the disease is misdiagnosed as malignant lymphoma in upto one-third of cases. Advances in diagnostic modalities including histopathology, immunohistochemistry, flow cytometry and molecular studies, have decreased incidence of misdiagnosis worldwide but, in our part of the world, it is still frequently misdiagnosed as probably, there remains lack of knowledge among physicians about the disease.

The common presentation of disease is with enlarged cervical lymph nodes and fever. Enlarged lymph nodes may be tender, and fever may be of low grade, sometimes associated with upper respiratory symptoms. Less common associations are arthralgia, skin rashes, fatigue, malaise, weight loss, decreased appetite, night sweats, diarrhea, nausea, vomiting, chest or abdominal pain and hepatosplenomegaly. Histologic findings include paracortical areas of coagulative necrosis with abundant karyorrhectic debris. Karyorrhectic foci are surrounded by histiocytes, plasmacytoid monocytes, immunoblasts and small and large lymphocytes, with abundance of T cells with predominance ofCD8+ overCD4+ Tcells. In this case, patient presented with high grade fever and lymphadenopathy. On clinical grounds, keeping endemic nature of TB in view with positive contact history and lack of response to more conservative treatment, she was misdiagnosed as TB lymphadenitis and was started on ATT. Her biopsy and histopathology was reviewed multiple times to make sure that the picture of histopathology is not consistent with TB. The patient was started on oral steroids and responded well. The clinicians are suggested to consider KFD as an important differential diagnosis in cervical lymphadenitis and fever, as its treatment is entirely different from that of TB.
KFD is a rare disease of benign nature. It is usually self-limited with excellent prognosis. It should be considered as possible differential of lymphadenopathy, particularly in settings where patient is not responding to ongoing treatment. Considering KFD as a differential, it may help physicians to avoid inappropriate use of ATT and may help avoid expensive work-up.

\section{PATIENT'S CONSENT:}

Informed consent was obtained from the patient to publish this case.

\section{CONFLICT OF INTEREST:}

Authors declared no conflict of interest.

\section{AUTHORS' CONTRIBUTION:}

SS: Searched the literature and has written the whole case report.

HM: Helped in literature review and discussion writing.

AM: Highlighted the importance of case, suggested to work upon this case and then helped in details writing of case scenario.

\section{REFERENCES}

1. Barbat B, Jhaj R, Khurram D. Fatality in Kikuchi-Fujimoto disease: A rare phenomenon. World J Clin Cases 2017; 5(2):35-39. doi:10.12998/wjcc.v5.i2.35.

2. Bosch X, Guilabert A, Miquel R, Campo E. Enigmatic Kikuchi-Fujimoto disease: A comprehensive review. Am J Clin Pathol 2004; 122:141-52. doi: 10.1309/YF08-1L4TKYWV-YVPQ.

3. Hino H, Nishimura T, Nitadori J, Miyakoshi S, Seki A, Arai T, et al. An uncommon presentation of Kikuchi-Fujimoto disease as mediastinal lymphadenopathy. J Thorac Dis 2016; 8(5):E330-E333. doi:10.21037/jtd.2016.03.78.

4. Ifeacho S, Aung T, Akinsola M. Kikuchi-Fujimoto disease: A case report and review of the literature. Cases J 2008; 1:187.

5. [5]Deaver D, Horna P, Cualing H, Sokol L. Pathogenesis, diagnosis, and management of Kikuchi-Fujimoto disease. Cancer Control 2014; 21:313-21.

6. Bogusz AM, Bhargava P. Recurrent histiocytic necrotizing lymphadenitis with a long latency in a patient with autoimmunity: a case report and review of literature. Int J Surg Pathol 2013; 21(3): 287-96. doi: 10.1177/ 1066896912467369 